\title{
No association between TNFSF15 and IL23R with ulcerative colitis in Koreans
}

\author{
Suk-Kyun Yang ${ }^{1}$, Yusun Jung ${ }^{2}$, Myunghee Hong ${ }^{2}$, Hyeri Kim² ${ }^{2}$, Byong Duk Ye ${ }^{1}$, Inchul Lee ${ }^{3}$ \\ and Kyuyoung Song ${ }^{2}$
}

\begin{abstract}
Recent genome-wide association studies have shown that some Crohn's disease (CD)-associated loci also contribute to ulcerative colitis (UC) susceptibility. To understand the genetic relationship between the two forms of inflammatory bowel disease, we investigated the well-established CD-susceptibility genes TNFSF15 (tumor necrosis factor ligand superfamily, member 15) and IL23R in Korean patients with UC. Eight TNFSF15 and five IL23R single-nucleotide polymorphisms were genotyped in 654 patients with UC and in 601 healthy controls. There was no association between TNFSF15 or IL23R variants and UC in Korean individuals, in contrast to previous reports of a shared association of the IL23R gene with both CD and UC in Caucasian individuals. Our data suggest that neither TNFSF15 nor IL23R variants contribute to UC susceptibility in Koreans. Journal of Human Genetics (2011) 56, 200-204; doi:10.1038/jhg.2010.166; published online 13 January 2011
\end{abstract}

Keywords: association; IL23R; Korean; TNFSF15; ulcerative colitis

\section{INTRODUCTION}

Ulcerative colitis (UC) and Crohn's disease (CD) are the two major forms of chronic inflammatory bowel diseases (IBD) of unknown etiology. Genetic epidemiology studies have suggested that UC and CD are polygenic diseases related to each other, and that genetic susceptibility and environmental factors are involved in the pathogenesis of both the diseases. ${ }^{1-4}$ The incidence of IBD is relatively higher in Caucasian than in Asian populations; however, the incidence in Asia is increasing. ${ }^{5,6}$ Of the two types of IBD, the genetic contribution to disease risk has been documented more extensively and clearly for $\mathrm{CD}^{7-17}$ than for UC. ${ }^{18-21}$ So far, linkage and genome-wide association studies have identified more than $30 \mathrm{CD}$-susceptibility loci in Caucasian populations. ${ }^{22}$ Of those, NOD2, ATG16L1 and IRGM were CD specific, whereas genes involved in Th17 differentiation, including IL23R, IL12B, JAK2, STAT3 and TNFSF15 were shared between UC and CD in Caucasians. ${ }^{23-26}$

TNFSF15 (tumor necrosis factor ligand superfamily, member 15) was the first CD-susceptibility gene identified through a genome-wide association screening of 72738 single-nucleotide polymorphisms (SNPs) in Japanese population. ${ }^{10}$ The association was well replicated by several studies, including our previous study in a Korean cohort, ${ }^{27}$ an independent Japanese cohort ${ }^{28}$ and a US cohort, ${ }^{29}$ and metaanalyses of genome-wide association studies in European populations, ${ }^{22}$ although the association was less significant in Europeans than in Japanese or Korean individuals. ${ }^{29,30}$ However, reports of an association of TNFSF15 with UC in Caucasians have been inconsistent. ${ }^{24-26}$

In a previous report on Korean patients with $\mathrm{CD}$, we replicated findings from studies on Caucasian populations, establishing inter- leukin 23 receptor (IL23R) and TNFSF15 as CD-susceptibility genes. ${ }^{27,31}$ Because some CD candidate genes involved in the Th17 pathway were shown to be shared with UC in Caucasians, in this study we sought to test the association between IL23R or TNFSF15 polymorphisms and UC in Koreans. We found that IL23R, which was reported to be associated with UC as well as CD in Caucasians, was not associated with UC in Koreans. However, TNFSF15 showed a marginal association with UC in male patients only.

\section{SUBJECTS AND METHODS}

Study subjects

The study population consisted of 654 UC patients ( 341 men and 313 women), $651 \mathrm{CD}$ patients (442 men and 209 women) and 601 unrelated healthy controls ( 310 men and 291 women). Part of this cohort has been studied previously. ${ }^{27,31}$ All patients with UC or CD were diagnosed at the IBD Clinic of Asan Medical Center, Seoul, Korea on the basis of conventional, clinical, radiological, endoscopic and histopathological criteria. ${ }^{32,33}$ Patients with indeterminate colitis were excluded from the study. For controls, disease-free individuals were recruited from the Health Promotion Center of the Asan Medical Center where routine health checkups are carried out. Phenotypic subgroups of UC were determined by two independent investigators (SKY and BDY), blinded to the results of the genotype analysis, using the Montreal classification. ${ }^{34}$ Briefly, phenotypes were classified on the basis of maximal endoscopic extent as E1 (ulcerative proctitis), E2 (left-sided UC) or E3 (extensive UC). The demographic and clinical characteristics of the study population are summarized in Table 1.

${ }^{1}$ Department of Gastroenterology, Asan Medical Center, University of Ulsan College of Medicine, Seoul, Korea; ${ }^{2}$ Department of Biochemistry and Molecular Biology, Asan Medical Center, University of Ulsan College of Medicine, Seoul, Korea and ${ }^{3}$ Department of Pathology, Asan Medical Center, University of Ulsan College of Medicine, Seoul, Korea Correspondence: Dr K Song, Department of Biochemistry and Molecular Biology, Asan Medical Center, University of Ulsan College of Medicine, 388-1 Pungnap-Dong, Songpa-Gu, Seoul 138-736, Korea.

E-mail: kysong@amc.seoul.kr

Received 14 September 2010; revised 9 November 2010; accepted 3 December 2010; published online 13 January 2011 
Table 1 Clinical characteristic of the study subjects

\begin{tabular}{lccc}
\hline & $\begin{array}{c}\text { U }(\mathrm{n}=654) \\
N(\%)\end{array}$ & $\begin{array}{c}C D(\mathrm{n}=651) \\
N(\%)\end{array}$ & $\begin{array}{c}\text { Controls }(\mathrm{n}=601) \\
N(\%)\end{array}$ \\
\hline Sex & & & \\
$\quad$ Male & $341(52.1)$ & $442(67.9)$ & $310(51.6)$ \\
$\quad$ Female & $313(47.9)$ & $209(32.1)$ & $291(48.4)$ \\
Mean age at sampling (year) & $40.8 \pm 13.3$ & $27.4 \pm 8.1$ & $30.7 \pm 11.2$ \\
Mean age at diagnosis (year) & $36.7 \pm 12.9$ & $24.3 \pm 7.7$ & \\
& & & \\
Extent & & & \\
Proctitis & $199(30.4)$ & & \\
Left-sided colitis & $204(31.2)$ & & \\
Extensive colitis & $251(38.4)$ & & \\
\hline
\end{tabular}

Abbreviations: $\mathrm{CD}$, Crohn's disease; UC, ulcerative colitis.

Gender distributions were statistically similar in UC and control groups, but age distributions were not. This study was approved by the institutional review board of Asan Medical Center, and written informed consent was obtained from all the subjects.

\section{SNP genotyping}

Genomic DNA was isolated from 5-ml samples of EDTA-anticoagulated venous blood by standard methods, using proteinase $\mathrm{K}$ and phenol/ chloroform extraction. Eight TNFSF15 SNPs (rs4574921, rs10114470, rs3810936, rs6478108, rs4979462, rs7848647, rs7865494 and rs1322057) and five IL23R SNPs (rs1004819, rs2201841, rs10889677, rs11209032 and rs1495965) were selected for genotyping. Samples were genotyped using a matrix-assisted laser desorption/ionization time-of-flight mass spectrometry-based system (Sequenom, San Diego, CA, USA) at the Analytical Genetics Technology Centre, Princess Margaret Hospital/ University Health Network in Toronto, Canada.

\section{Statistical analysis}

Genotype frequencies between cases and controls were checked separately for consistency with those expected from Hardy-Weinberg equilibrium using SNP Alyze software, version 5.0 (Dynacom, Yokohama, Japan). The associations were examined by comparing allele and genotype frequencies in different groups of subjects using a $\chi^{2}$ test with permutation correction using PLINK 2.050 (http://pngu.mgh. harvard.edu/purcell/plink/) $)^{35}$ and version 4.2 of the Haploview program (http://www.broadinstitute.org/haploview/haploview). Allelic frequencies were compared between cases and controls using logistic regression to calculate age- and gender-adjusted odds ratios and 95\% confidence intervals. Genotype-phenotype analyses were performed for SNPs in cases in which the $P$-value was less than 0.2 in case-control analysis. Logistic regression analyses were also conducted to examine any significant associations between polymorphisms and disease phenotypes. The data were analyzed using SPSS version 18.0.0 for windows (SPSS, Chicago, IL, USA).

\section{RESULTS}

Association analysis of TNFSF15 and IL23R polymorphisms with UC in Koreans

Eight TNFSF15 SNPs and five IL23R SNPs were genotyped in 654 UC patients and 601 healthy controls. None of the 13 SNPs showed significant deviation from the Hardy-Weinberg equilibrium.

The genotypic and allelic distributions of the 13 SNPs are summarized in Table 2. A $\chi^{2}$ test of homogeneity in both allele and genotype distributions among UC patients and controls failed to show any

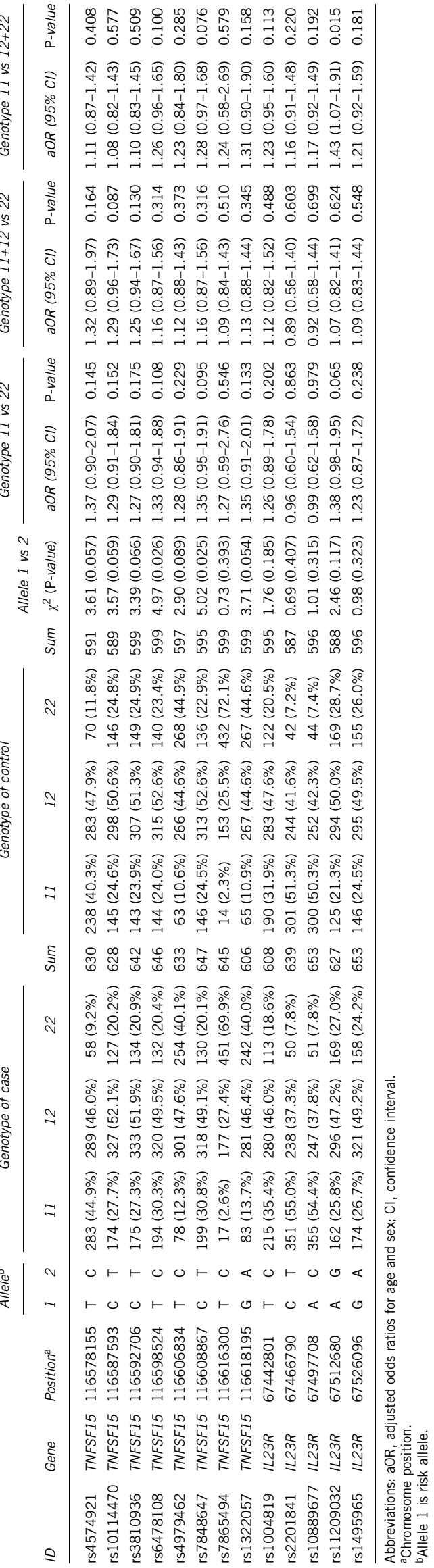

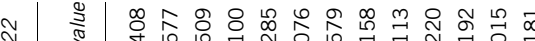
1. จ i $m$ b t $\begin{aligned} & 1 \\ & 1\end{aligned}$

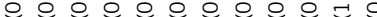
开

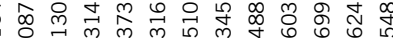

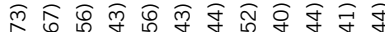

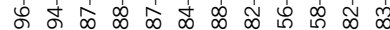

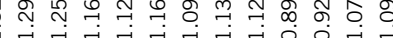

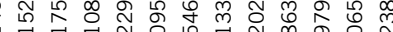

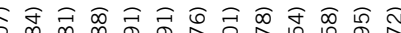

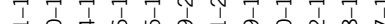

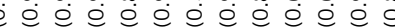
ণิ సิ कूष

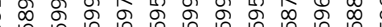

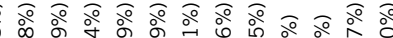

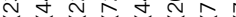
子

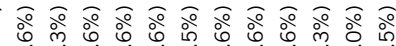

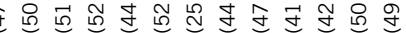

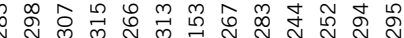

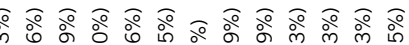

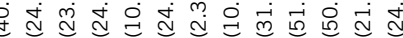

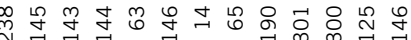

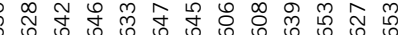

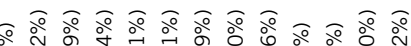
赵过

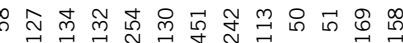

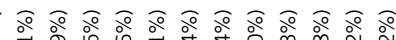

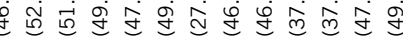

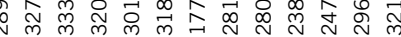
らㅇㅇㅇ

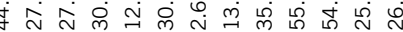

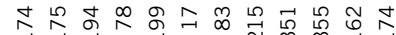
$0 \vdash \vdash 0010 \ll 0 \vdash 00 \ll$ O 0 - 
Table 3 Association of TNFSF15 between IBD cases and controls

\begin{tabular}{|c|c|c|c|c|c|c|c|c|c|c|c|}
\hline \multirow[b]{2}{*}{$I D$} & \multirow[b]{2}{*}{ Position } & \multicolumn{3}{|c|}{ Risk allele } & \multicolumn{4}{|c|}{$R A F$} & \multicolumn{3}{|c|}{ Association P-value } \\
\hline & & $I B D$ & $U C$ & $C D$ & Control & $I B D$ & $U C$ & $C D$ & $I B D$ & $U C$ & $C D$ \\
\hline rs10114470 & 116587593 & c & C & $\mathrm{C}$ & 0.499 & 0.614 & 0.537 & 0.692 & 4.3E-11 & 5.9E-02 & $3.8 \mathrm{E}-22$ \\
\hline rs3810936 & 116592706 & C & C & $\mathrm{C}$ & 0.495 & 0.613 & 0.532 & 0.694 & 7.9E-12 & $6.6 \mathrm{E}-02$ & $4.9 \mathrm{E}-24$ \\
\hline rs6478108 & 116598524 & $\mathrm{~T}$ & $\mathrm{~T}$ & $\mathrm{~T}$ & 0.503 & 0.636 & 0.548 & 0.724 & $1.0 \mathrm{E}-14$ & 2.6E-02 & $9.0 \mathrm{E}-30$ \\
\hline rs7865494 & 116616300 & $\mathrm{~T}$ & $T$ & $\mathrm{~T}$ & 0.151 & 0.158 & 0.164 & 0.153 & 5.7E-01 & 3.9E-01 & $8.9 \mathrm{E}-01$ \\
\hline rs1322057 & 116618195 & G & $\mathrm{G}$ & G & 0.331 & 0.467 & 0.369 & 0.565 & $6.7 \mathrm{E}-15$ & 5.4E-02 & $8.9 \mathrm{E}-31$ \\
\hline
\end{tabular}

Abbreviations: CD, Crohn's disease; IBD, inflammatory bowel disease; RAF, risk allele frequency; UC, ulcerative colitis.

Table 4 Association of IL23R between IBD cases and controls

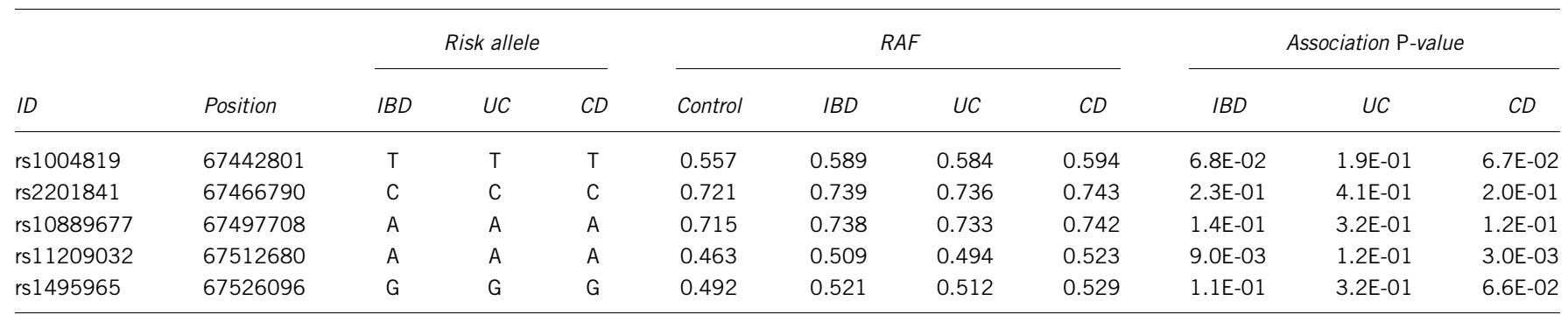

Abbreviations: CD, Crohn's disease; IBD, inflammatory bowel disease; RAF, risk allele frequency; UC, ulcerative colitis.

significant markers. The differences in allele frequency of the eight TNFSF15 SNPs between controls and UC patients ranged from $1.3 \%$ for rs7865494 to $4.5 \%$ for both rs6478108 and rs7848647. However, upon association analyses by gender, a few markers from TNFSF15 showed marginally significant association with UC in men only. Four markers showing marginally significant associations only in male UC patients include rs10114470 (5.5\%, $P=0.050)$, rs6478108 (7.0\%, $P=0.012)$, rs7848647 (6.9\%, $P=0.014)$ and $\operatorname{rs} 1322057 \quad(5.5 \%$, $P=0.044)$. The differences in allele frequencies of the four SNPs in women UC patients were 2.0, 1.7, 2.0 and $1.9 \%$, respectively, and were not statistically significant. The adjusted OR for rs10114470 in men was $1.55(P=0.032)$, for rs6478108 was $1.48(P=0.034)$ and for rs7848647 was $1.48(P=0.034)$. However, the significance of these results could not be confirmed using a 1000 permutation test.

A comparison of our UC data with our CD data (Table 3) showed that seven of the eight SNPs of TNFSF15 tested, showed significant associations with $\mathrm{CD}$. The differences in allele frequency of the eight TNFSF15 SNPs between controls and CD patients ranged from $16 \%$ for rs 4574921 to $23.4 \%$ for rs 1322057 . The adjusted odds ratio of rs1322057 for GG genotype in comparison with AA genotype was 6.91 ( $95 \%$ confidence interval $\left.=4.81-9.92, P=1.5 \times 10^{-25}\right)$ and adjusted odds ratio of rs4574921 for CC genotype in comparison with TT genotype was 4.65 (95\% confidence interval=2.82-7.68, $P=1.9 \times 10^{-9}$ ).

In the five IL23R SNPs genotyped in the Korean samples, the differences in the risk allele frequency between the UC and control groups were $2.7 \%$ for $\mathrm{rs} 1004819,1.5 \%$ for $\mathrm{rs} 2201841,1.8 \%$ for rs10889677, 3.1\% for rs 11209032 and $2.0 \%$ for rs1495965; none of these differences were statistically significant. Following association analyses, none of them showed significant association with UC (Table 2). However, in CD patients, the differences in the risk allele frequency between the $\mathrm{CD}$ and control groups were $3.7 \%$ for rs1004819, 6.0\% for rs11209032 and 3.7\% for rs1495965, and were statistically significant (Table 4). The three SNPs (rs1004819, rs11209032 and rs1495965) showed significant associations with CD ( $P=0.003$ in a recessive model for rs1004819; $P=0.003$ in an allelic model for rs11209032; $P=0.031$ in a recessive model for rs1495965).

\section{DISCUSSION}

Recent studies have shown that genes involved in Th17 differentiation, including IL23R, IL12B, JAK2, STAT3, CCR6 and TNFSF15, are associated with both $\mathrm{CD}$ and UC in Caucasians. ${ }^{23-26}$ In the present study, we investigated the well-established CD-susceptibility genes TNFSF15 and IL23R in Korean UC patients.

TNFSF15 is a CD-susceptibility gene in Asians as well as in Caucasian individuals. ${ }^{10,16,27-30}$ In fact, the association of TNFSF15 with $\mathrm{CD}$ is much stronger in Asians than in Caucasians..$^{29,30}$ The association of $I L 23 R$ with CD is consistently observed in Caucasians, but among Asians, this association has been demonstrated only in Koreans. ${ }^{31}$ Given that both IL23R and TNFSF15 were suggested to be associated with UC in Caucasians, ${ }^{11,25,26,29}$ we sought to test the association between polymorphisms of IL23R and/or TNFSF15 and UC in Koreans.

We failed to observe a significant association of TNFSF15 polymorphisms with UC in Korean individuals, except marginal association with male UC patients. Previous reports of TNFSF15 association with UC in Caucasians have been inconsistent, ${ }^{24-26}$ although they did not test identical SNPs of TNFSF15. Franke et al. ${ }^{24}$ reported an 
association of rs7869487 of TNFSF15 with CD and IBD $(P=0.010$, $P=0.031$, respectively) but not with UC $(P=0.42)$, whereas Wang et al. ${ }^{25}$ reported a significant association of rs4263839 with UC $\left(P=8.6 \times 10^{-4}\right)$. Recently, it was reported in a genome-wide association study that rs 11554257 located $39.2 \mathrm{~kb}$ upstream of TNFSF15 was implicated in severe UC, as medically refractory cases were compared with healthy controls $\left(P<5.0 \times 10^{-5}\right) .^{26}$ Interestingly, rs7869487, which failed to show an association with UC in Caucasians, is located $12.5 \mathrm{~kb}$ upstream of TNFSF15 and $2.5 \mathrm{~kb}$ from rs1322057, which showed the most significant association with $\mathrm{CD}\left(P=1.5 \times 10^{-25}\right.$, $\mathrm{OR}=6.91$ ), but not with UC, in our Korean population. rs4263839 in intron 1 of TNFSF15 showed a significant association with UC in Caucasians, but rs4979462, 574 bp away from rs4263839, showed strong association with CD only in Koreans. Although our study was ongoing, a Japanese group reported an association of TNFSF15 with UC and CD. ${ }^{36}$ This study tested a single SNP from TNFSF15 (rs3810936) in small groups of 82 UC patients, 130 CD patients and 163 controls, and reported positive associations with both UC and CD. However, in our study on Koreans, the difference in the frequency of this allele between controls and UC patients was only $3.7 \%$ in comparison with $11.2 \%$ in the Japanese population, and was not statistically significant. We also investigated the association of TNFSF15 polymorphism with UC sub-phenotypes based on disease extent, but did not find any significant association between specific phenotypes and TNFSF15 variants.

We failed to replicate an association of IL23R with UC in Koreans. Although none of the five IL23R SNPs showed a positive association with UC, rs1004819, rs11209032 and rs1495965 showed significant associations with CD in Koreans. Upon a subtype analysis of UC, a statistically marginal association was observed between left-sided colitis and IL23R SNPs rs10889677 ( $P=0.022$ in a dominant model) and rs2201841 $(P=0.029$ in a genotypic model) (data not shown). Notably, the SNPs of IL23R that showed an association with CD or subtypes of UC are not overlapping. Our samples represent one of the largest IBD collections from a single institution under strict inclusion criteria. However, it is possible that our subgroup analyses are underpowered because of the limited sample size for revealing the genotypephenotype relationship. Therefore, it is necessary to expand the number of samples of each UC subgroup for further studies. We also need to expand our study to include other genes implicated in Th17 differentiation than TNFSF15 and IL23R to investigate the differential pathogenesis of UC and CD in Asians.

In summary, we report no significant association between TNFSF15 or IL23R polymorphisms and UC in a Korean population.

\section{ACKNOWLEDGEMENTS}

This work was supported by a grant to K Song (A090039) by the Korea Healthcare Technology R\&D Project, MOHW (Ministry of Health, Welfare \& Family Affairs), Republic of Korea and a grant to S-K Yang by Ferring Pharmaceuticals Korea.

1 Xavier, R. J. \& Podolsky, D. K. Unravelling the pathogenesis of inflammatory bowel disease. Nature 448, 427-434 (2007).

2 Tysk, C., Lindberg, E., Jarnerot, G. \& Floderus-Myrhed, B. Ulcerative colitis and Crohn's disease in an unselected population of monozygotic and dizygotic twins. A study of heritability and the influence of smoking. Gut 29, 990-996 (1988)

3 Orholm, M., Munkholm, P., Langholz, E., Nielsen, O. H., Sørensen, T. I. \& Binder, V. Familial occurrence of inflammatory bowel disease. N. Engl. J. Med. 324, 84-88 (1991).

4 Loftus, E. V. Jr Clinical epidemiology of inflammatory bowel disease: incidence, prevalence, and environmental influences. Gastroenterology 126, 1504-1517 (2004).
5 Thia, K. T., Loftus, E. V. Jr, Sandborn, W. J. \& Yang, S.- K. An update on the epidemiology of inflammatory bowel disease in Asia. Am. J. Gastroenterol. 103, 3167-3182 (2008)

6 Yang, S.- K., Loftus, E. V. Jr \& Sandborn, W. J. Epidemiology of inflammatory bowel disease in Asia. Inflamm. Bowel Dis. 7, 260-270 (2001).

7 Hampe, J., Cuthbert, A., Croucher, P. J., Mirza, M. M., Mascheretti, S., Fisher, S. et al. Association between insertion mutation in NOD2 gene and Crohn's disease in German and British populations. Lancet. 357, 1925-1928 (2001).

8 Hugot, J.- P., Chamaillard, M., Zouali, H., Lesage, S., Cézard, J. P., Belaiche, J. et al. Association of NOD2 leucine-rich repeat variants with susceptibility to Crohn's disease. Nature 411, 599-603 (2001).

9 Ogura, Y., Bonen, D. K., Inohara, N., Nicolae, D. L., Chen, F. F., Ramos, R. et al. A frameshift mutation in NOD2 associated with susceptibility to Crohn's disease. Nature 411, 603-606 (2001).

10 Yamazaki, K., McGovern, D., Ragoussis, J., Paolucci, M., Butler, H., Jewell, D. et al. Single nucleotide polymorphisms in TNFSF15 confer susceptibility to Crohn's disease. Hum. Mol. Genet. 14, 3499-3506 (2005).

11 Duerr, R. H., Taylor, K. D., Brant, S. R., Rioux, J. D., Silverberg, M. S., Daly, M. J. et al. A genome-wide association study identifies IL23R as an inflammatory bowel disease gene. Science 314, 1461-1463 (2006).

12 Rioux, J. D., Xavier, R. J., Taylor, K. D., Silverberg, M. S., Goyette, P., Huett, A. et al. Genome-wide association study identifies new susceptibility loci for Crohn's disease and implicates autophage in disease pathogenesis. Nat. Genet. 39, 596-604 (2007).

13 Hampe, J., Franke, A., Rosenstiel, P., Till, A., Teuber, M., Huse, K. et al. A genome-wide association scan of nonsynonymous SNPs identifies a susceptibility variant for Crohn's disease in ATG16L1. Nat. Genet. 39, 207-211 (2007).

14 Libioulle, C., Louis, E., Hansoul, S., Sandor, C., Farnir, F., Franchimont, D. et al. Novel Crohn's disease locus identified by genome-wide association maps to a gene desert on 5p13.1 and modulates expression of PTGER4. PLoS Genet. 3, e58 (2007).

15 Franke, A., Hampe, J., Rosenstiel, P., Becker, C., Wagner, F., Häsler, R. et al. Systemic association mapping identifies NELL1 as a novel IBD disease gene. PLoS One. 2, e691 (2007).

16 The Wellcome Trust Case Control Consortium. Genome-wide association study of 14000 cases of seven common diseases and 3000 shared controls. Nature 447, 661-678 (2007).

17 Parkes, M., Barrett, J. C., Prescott, N. J., Tremelling M., Anderson, C. A., Fisher, S. A et al. Sequence variants in the autophage gene IRGM and multiple other replicating loci contribute to Crohn's disease susceptibility. Nat. Genet. 39, 830-832 (2007).

18 Fisher, S. A., Tremelling, M., Anderson, C. A., Gwilliam, R., Bumpstead, S., Prescott, N. J. et al. Genetic determinants of ulcerative colitis include the ECM 1 locus and five loci implicated in Crohn's disease. Nat. Genet. 40, 710-712 (2008).

19 Franke, A., Balschun, T., Karlsen, T. H., Sventoraityte, J., Nikolaus, S., Mayr, G. et al. Sequence variants in IL10, ARPC2 and multiple other loci contribute to ulcerative colitis susceptibility. Nat. Genet. 40, 1319-1323 (2008).

20 Asano, K., Matsushita, T., Umeno, J., Hosono, N., Takahashi, A., Kawaguchi, T. et al. A genome-wide association study identifies three new susceptibility loci for ulcerative colitis in the Japanese population. Nat. Genet. 41, 1325-1329 (2009).

21 Silverberg, M. S., Cho, J. H., Rioux, J. D., McGovern, D. P., Wu, J., Annese, V. et al. Ulcerative colitis-risk loci on chromosomes $1 \mathrm{p} 36$ and $12 \mathrm{q} 15$ found by genome-wide association study. Nat. Genet. 41, 216-220 (2009).

22 Barrett, J. C., Hansoul, S., Nicolae, D. L., Cho, J. H., Duerr, R. H., Rioux, J. D. et al. Genome-wide association defines more than 30 distinct susceptibility loci for Crohn's disease. Nat. Genet. 40, 955-962 (2008).

23 Anderson, C. A., Massey, D. C., Barrett, J. C., Prescott, N. J., Tremelling, M., Fisher, S. A. et al. Investigation of Crohn's disease risk loci in ulcerative colitis further defines their molecular relationship. Gastroenterology 136, 523-529 (2009).

24 Franke, A., Balschun, T., Karlsen, T. H., Hedderich, J., May, S., Lu, T. et al. Replication of signals from recent studies of Crohn's disease identifies previously unknown disease loci for ulcerative colitis. Nat. Genet. 40, 713-715 (2008).

25 Wang, K., Baldassano, R., Zhang, H., Qu, H. Q., Imielinski, M., Kugathasan, S. et al. Comparative genetic analysis of inflammatory bowel disease and type 1 diabetes implicates multiple loci with opposite effects. Hum. Mol. Genet. 19, 2059-2067 (2010).

26 Haritunians, T., Taylor, K., Targan, S. R., Dubinsky, M., Ippoliti, A., Kwon, S. et al. Genetic predictors of medically refractory ulcerative colitis. Inflamm. Bowel Dis. 16, 1830-1840 (2010)

27 Yang, S.- K., Lim, J., Chang, H. S., Lee, I., Li, Y., Liu, J. et al. Association of TNFSF15 with Crohn's disease in Koreans. Am. J. Gastroenterol. 103, 1437-1442 (2008).

28 Kakuta, Y., Kinouchi, Y., Negoro, K., Takahashi, S. \& Shminosegawa, T. Association study of TNFSF15 polymorphisms in Japanese patients with inflammatory bowel disease. Gut 55, 1527-1528 (2006).

29 Picornell, Y., Mei, L., Taylor, K., Yang, H., Targan, S. R. \& Rotter, J. I. TNFSF15 is an ethnic-specific IBD gene. Inflamm. Bowel Dis. 13, 1333-1338 (2007).

30 Cho, J. H. \& Weaver, C. T. The genetics of inflammatory bowel disease. Gastroenterology 133, 1327-1339 (2007)

31 Yang, S.- K., Park, M., Lim, J., Park, S. H., Ye, B. D., Lee, I. et al. Contribution of IL23R but not ATG16L1 to Crohn's disease susceptibility in Koreans. Inflamm. Bowel Dis. 15, 1385-1390 (2009).

32 Lennard-Jones, J. E. Classification of inflammatory bowel disease. Scand. J. Gastroenterol. Suppl. 170, 2-6 (1989). 
33 Park, S. H., Kim, Y. M., Yang, S.- K., Kim, S. H., Byeon, J. S., Myung, S. J. et al. Clinical features and natural history of ulcerative colitis in Korea. Inflamm. Bowel Dis. 13, 278-283 (2007).

34 Silverberg, M. S., Satsangi, J., Ahmad, T., Arnott, I. D., Bernstein, C. N., Brant, S. R. et al. Toward an integrated clinical, molecular and serological classification of inflammatory bowel disease: Report of a Working Party of the 2005 Montreal World Congress of Gastroenterology. Can. J. Gastroenterol. 19(Suppl A), 5-36 (2005).
35 Purcell, S., Neale, B., Todd-Brown, K., Thomas, L., Ferreira, M. A., Bender, D. et al. PLINK: a tool set for whole-genome association and population-based linkage analyses. Am. J. Hum. Genet. 81, 559-575 (2007).

36 Nakagome, S., Takeyama, Y., Mano, S., Sakisaka, S., Matsui, T., Kawamura, S. et al. Population-specific susceptibility to Crohn's disease and ulcerative colitis; dominant and recessive relative risks in the Japanese population. Ann. Hum. Genet. 74, 126-136 (2010). 